\title{
A Method for Stability Analysis of Periodic Delay Differential Equations with Multiple Time-Periodic Delays
}

\author{
Gang Jin, ${ }^{1,2}$ Houjun Qi, ${ }^{1,2}$ Zhanjie Li, ${ }^{1,2}$ Jianxin Han, ${ }^{1,2}$ and Hua $\mathrm{Li}^{3}$ \\ ${ }^{1}$ Tianjin Key Laboratory of High Speed Cutting and Precision Machining, Tianjin University of Technology and Education, \\ Tianjin 300222, China \\ ${ }^{2}$ National-Local Joint Engineering Laboratory of Intelligent Manufacturing Oriented Automobile Die \& Mould, \\ Tianjin University of Technology and Education, Tianjin 300222, China \\ ${ }^{3}$ Tianjin Jinhang Institute of Technical Physics, Tianjin, China
}

Correspondence should be addressed to Jianxin Han; hanjianxin@tju.edu.cn

Received 28 June 2017; Accepted 14 August 2017; Published 17 September 2017

Academic Editor: Renming Yang

Copyright (C) 2017 Gang Jin et al. This is an open access article distributed under the Creative Commons Attribution License, which permits unrestricted use, distribution, and reproduction in any medium, provided the original work is properly cited.

Delay differential equations (DDEs) are widely utilized as the mathematical models in engineering fields. In this paper, a method is proposed to analyze the stability characteristics of periodic DDEs with multiple time-periodic delays. Stability charts are produced for two typical examples of time-periodic DDEs about milling chatter, including the variable-spindle speed milling system with one-time-periodic delay and variable pitch cutter milling system with multiple delays. The simulations show that the results gained by the proposed method are in close agreement with those existing in the past literature. This indicates the effectiveness of our method in terms of time-periodic DDEs with multiple time-periodic delays. Moreover, for milling processes, the proposed method further provides a generalized algorithm, which possesses a good capability to predict the stability lobes for milling operations with variable pitch cutter or variable-spindle speed.

\section{Introduction}

Time-delay systems widely exist in engineering and science, where the rate of change of state is determined by both present and past state variables, such as machining processes $[1,2]$, wheel dynamics $[3,4]$, feedback controller $[5,6]$, gene expression dynamics [7], and population dynamics [8,9]. However, for some of above applications, the time delay in the dynamic system may lead to instability, poor performance, or other types of potential damage. Therefore, it is necessary for engineers and scientists to research the dynamics of these systems to reduce or avoid such problems.

Compared to the finite dimensional dynamics for systems without time delay, time-delay systems have infinitedimensional dynamics and are usually described by delay differential equations (DDEs). Their stability properties can be analyzed through obtaining the stability charts that show the stable and unstable domains. For example, a stable milling process can be realized by choosing the corresponding parameter from a stability lobe diagram (SLD), which is a function of spindle speed and depth of cut parameters. Thus, more and more attention has been paid on this issue and many analytical and numerical methods have been developed to derive the stability conditions for the system parameters.

By using the D-subdivision method, Bhatt and Hsu [10] determined stability criteria for second-order scalar DDEs. Budak and Altıntaş [11, 12] and Merdol and Altintas [13] proposed a method in frequency domain called multifrequency solution. By employing a shifted Chebyshev polynomial approximation, Butcher et al. $[14,15]$ presented a new technique to study the stability properties of dynamic systems by obtaining an approximate monodromy matrix. Insperger and Stepan [16-18] proposed a known method called semidiscretization method (SDM), which is based on the discretization of the DDEs and approximates their infinite-dimensional phase space by a finite discrete map in time domain. Bayly et al. [19] carried out a temporal finite element analysis for solving the DDEs, which are written in 
the form of a state space model and discretizing the time interval of interest into a finite number of temporal elements. Based on the direct integration scheme, Ding et al. [20], Liu et al. [21], and Jin et al. [22] used a full-discretization method to gain stability chart efficiently. Recently, Khasawneh and Mann [23] and Lehotzky et al. [24] presented a numerical algorithm called spectral element method. This method has good efficiency because of its highly accurate numerical quadratures for the integral terms.

SDM is a known and widely used method to determine stability charts for general time-periodic DDEs arising in different engineering problems. In this paper, based on SDM, a generalized method for periodic DDEs with multiple timeperiodic delays is proposed to obtain the stability chart of DDEs. The structure of the paper is as follows. In Section 2, the mathematical model is introduced. In Section 3, two typical examples are used to verify the effectiveness of the proposed method. In Section 4, conclusions with a brief discussion are presented.

\section{Mathematical Model}

The general form of linear, time-periodic DDEs with multiple time-periodic delays can be expressed as

$$
\begin{aligned}
& \dot{\mathbf{y}}(t)=\mathbf{A}_{0} \mathbf{y}(t)+\mathbf{A}(t) \mathbf{y}(t)-\sum_{j=1}^{N} \mathbf{B}_{j}(t) \mathbf{u}\left(t-\tau_{j}(t)\right) \\
& \mathbf{u}(t)=\mathbf{D} \mathbf{y}(t),
\end{aligned}
$$

where $\mathbf{y}(t) \in \mathbb{R}^{n}$ is the state, $\mathbf{u}(t) \in \mathbb{R}^{m}$ is the input, $\mathbf{A}_{0}$ is a constant matrix, $\mathbf{A}(t)$ and $\mathbf{B}_{j}(t)$, respectively, are $n \times n$ and $n \times m$ periodic coefficient matrices that satisfy $\mathbf{A}(t)=\mathbf{A}(t+T)$ and $\mathbf{B}_{j}(t)=\mathbf{B}_{j}(t+T), j=1,2, \ldots, N$, and $\tau_{j}(t)=\tau_{j}(t+T)>$ $0, \mathbf{D}$ is an $m \times n$ constant matrix, $T$ is the time period, and $N$ is the number of time-delays. Note that (1) can also be written in the form

$$
\dot{\mathbf{y}}(t)=\mathbf{A}_{0} \mathbf{y}(t)+\mathbf{A}(t) \mathbf{y}(t)-\sum_{j=1}^{N} \mathbf{C}_{j}(t) \mathbf{y}\left(t-\tau_{j}(t)\right)
$$

with $\mathbf{C}_{j}(t)=\mathbf{B}_{j}(t) D$.

Consider that the period $T$ is divided into $k$ number of discrete time intervals, such that each interval length $\Delta t=$ $T / k$. Introduce symbol $\left[t_{i}, t_{i+1}\right]$ to represent the $i$ th time interval, $i \in \mathbb{Z}^{+}$. Here, $t_{i}$ means the $i$ th time node and is equal to $i \Delta t$. Thus, considering the idea in [2], the averaged delay for the discretization interval $\left[t_{i}, t_{i+1}\right]$ is defined as follows:

$$
\tau_{i, j}=\frac{1}{\Delta t} \int_{t_{i}}^{t_{i+1}} \tau_{j}(t) d t=\tau_{0, j}-\tau_{1, j} c_{i},
$$

where

$$
c_{i}=\frac{k}{2 \pi} \int_{i 2 \pi / k}^{(i+1) 2 \pi / k} \sin (t) d t, \quad i=0,1, \ldots, k-1 .
$$

The number of intervals $m_{i, j}$ related to the delay item $\tau_{i, j}$ can be approximately obtained by

$$
m_{i, j}=\operatorname{int}\left(\frac{\tau_{i, j}+\Delta t / 2}{\Delta t}\right),
$$

where int $(*)$ indicates the operation that rounds positive number towards zero.

Substituting (3) into (2) and solving it as an ordinary differential equation over the discretization period $\left[t_{i}, t_{i+1}\right]$ with initial condition $x\left(t_{i}\right)=x_{i}$, the following equation is derived:

$$
\begin{aligned}
\mathbf{y}(t) & =e^{\mathbf{A}_{0}\left(t-t_{i}\right)} \mathbf{y}_{i}+\int_{t_{i}}^{t}\left\{e^{\mathbf{A}_{0}(t-\xi)}[\mathbf{A}(\xi) \mathbf{y}(\xi)\right. \\
& \left.\left.-\sum_{j=1}^{N} \mathbf{C}_{j}(\xi) \mathbf{y}\left(\xi-\tau_{i, j}\right)\right]\right\} d \xi .
\end{aligned}
$$

Substituting $t=t_{i+1}$ into (6), then it can be equivalently expressed as

$$
\begin{aligned}
& \mathbf{y}\left(t_{i+1}\right)=e^{\mathbf{A}_{0} \Delta t} \mathbf{y}_{i}+\int_{t_{i}}^{t_{i+1}}\left\{e^{\mathbf{A}_{0}\left(t_{i+1}-\xi\right)}[\mathbf{A}(\xi) \mathbf{y}(\xi)\right. \\
& \left.\left.-\sum_{j=1}^{N} \mathbf{C}_{j}(\xi) \mathbf{y}\left(\xi-\tau_{i, j}\right)\right]\right\} d \xi
\end{aligned}
$$

In $\left[t_{i}, t_{i+1}\right], \mathbf{A}(t), \mathbf{C}_{j}(t), \mathbf{y}(t)$, and $\mathbf{y}\left(t-\tau_{i, j}\right)$ are defined as follows:

$$
\begin{gathered}
\mathbf{A}(t)=\mathbf{A}_{i}+\frac{\mathbf{A}_{i+1}-\mathbf{A}_{i}}{\Delta t}\left(t-t_{i}\right), \\
\mathbf{C}_{j}(t)=\mathbf{C}_{i, j}+\frac{\mathbf{C}_{i+1, j}-\mathbf{C}_{i, j}}{\Delta t}\left(t-t_{i}\right), \\
\mathbf{y}(t)=\mathbf{y}_{i}+\frac{\mathbf{y}_{i+1}-\mathbf{y}_{i}}{\Delta t}\left(t-t_{i}\right), \\
\mathbf{y}\left(t-\tau_{i, j}\right)=\beta_{i, j} \mathbf{y}_{i-m_{i, j}}+\alpha_{i, j} \mathbf{y}_{i+1-m_{i, j}},
\end{gathered}
$$

where $\mathbf{A}_{i}=\mathbf{A}\left(t_{i}\right), \mathbf{C}_{i, j}=\mathbf{C}_{j}\left(t_{i}\right), \mathbf{y}_{i}=\mathbf{y}\left(t_{i}\right), \alpha_{i, j}=\left(m_{i, j} \Delta t+\right.$ $\left.\Delta t / 2-\tau_{i, j}\right) / \Delta t$, and $\beta_{i, j}=1-\alpha_{i, j}$. Here, it should be noted that the approximation of $\mathbf{y}\left(t-\tau_{i, j}\right)$ in (8) is the same as that for the so-called zero-order SDM in [2].

Substituting (8) into (7) leads to

$$
\begin{aligned}
\mathbf{y}_{i+1}= & \left(\Phi_{0}+\mathbf{F}_{i}\right) \mathbf{y}_{i}+\mathbf{P}_{i} \mathbf{y}_{i+1} \\
& -\sum_{j=1}^{N}\left(\beta_{i, j} \mathbf{R}_{i, j} \mathbf{y}_{i-m_{i, j}}+\alpha_{i, j} \mathbf{R}_{i, j} \mathbf{y}_{i+1-m_{i, j}}\right),
\end{aligned}
$$

where

$$
\begin{aligned}
\mathbf{F}_{i}= & \left(\Phi_{1}-\frac{2}{\Delta t} \Phi_{2}+\frac{1}{\Delta t^{2}} \Phi_{3}\right) \mathbf{A}_{i} \\
& +\left(\frac{1}{\Delta t} \Phi_{2}-\frac{1}{\Delta t^{2}} \Phi_{3}\right) \mathbf{A}_{i+1} \\
\mathbf{P}_{i}= & \left(\frac{1}{\Delta t} \Phi_{2}-\frac{1}{\Delta t^{2}} \Phi_{3}\right) \mathbf{A}_{i}+\left(\frac{1}{\Delta t^{2}} \Phi_{3}\right) \mathbf{A}_{i+1}, \\
\mathbf{R}_{i, j}= & \left(\Phi_{1}-\frac{1}{\Delta t} \Phi_{2}\right) \mathbf{C}_{i, j}+\left(\frac{1}{\Delta t} \Phi_{2}\right) \mathbf{C}_{i+1, j} .
\end{aligned}
$$


Clearly, $\Phi_{0}, \Phi_{1}, \Phi_{2}$, and $\Phi_{3}$ can be expressed as follows:

$$
\begin{aligned}
& \Phi_{0}=e^{\mathbf{A}_{0} \Delta t}, \\
& \Phi_{1}=\int_{0}^{\Delta t} e^{\mathbf{A}_{0}(\Delta t-s)} d s=\mathbf{A}_{0}^{-1}\left(\Phi_{0}-\mathbf{I}\right), \\
& \Phi_{2}=\int_{0}^{\Delta t} s e^{\mathbf{A}_{0}(\Delta t-s)} d s=\mathbf{A}_{0}^{-1}\left(\Phi_{1}-\Delta t \mathbf{I}\right), \\
& \Phi_{3}=\int_{0}^{\Delta t} s^{2} e^{\mathbf{A}_{0}(\Delta t-s)} d s=\mathbf{A}_{0}^{-1}\left(2 \Phi_{2}-\Delta t^{2} \mathbf{I}\right),
\end{aligned}
$$

where $\mathbf{I}$ denotes the identity matrix. Let $M=\max \left(m_{i . j}\right)$ and

$$
\mathbf{Z}_{i}=\operatorname{col}\left(\mathbf{y}_{i}, \mathbf{y}_{i-1} \cdots \mathbf{y}_{i-M}\right) ;
$$

then combining (9) and (12), one can be recast into a discrete map as

$$
\mathbf{Z}_{i+1}=\mathbf{D}_{i} \mathbf{Z}_{i}
$$

where each $\mathbf{D}_{i}$ matrix is given by

$$
\begin{aligned}
& \mathbf{D}_{i}=\left[\begin{array}{cccccc}
\mathbf{H}_{i+1}\left(\Phi_{0}+\mathbf{F}_{i}\right) & 0 & \cdots & 0 & 0 & 0 \\
\mathbf{I} & 0 & \cdots & 0 & 0 & 0 \\
0 & \mathbf{I} & \cdots & 0 & 0 & 0 \\
\vdots & \vdots & \ddots & \vdots & \vdots & \vdots \\
0 & 0 & \cdots & \mathbf{I} & 0 & 0 \\
0 & 0 & \cdots & 0 & \mathbf{I} & 0
\end{array}\right] \\
& +\sum_{j=1}^{N}\left[\begin{array}{cccccc}
0 & \cdots & -\mathbf{H}_{i+1} \alpha_{i, j} \mathbf{R}_{i, j} & -\mathbf{H}_{i+1} \beta_{i, j} \mathbf{R}_{i, j} & \cdots & 0 \\
0 & \cdots & 0 & 0 & \cdots & 0 \\
0 & \cdots & 0 & 0 & \cdots & 0 \\
\vdots & \vdots & \ddots & \vdots & \vdots & \vdots \\
0 & \cdots & 0 & 0 & \cdots & 0 \\
0 & \cdots & 0 & 0 & \cdots & 0
\end{array}\right] \text {, }
\end{aligned}
$$

where $\mathbf{H}_{i+1}=\left(\mathbf{I}-\mathbf{P}_{i}\right)^{-1}$. The horizontal position of the discrete input matrices $\mathbf{H}_{i+1} \alpha_{i, j} \mathbf{R}_{i, j}$ and $\mathbf{H}_{i+1} \beta_{i, j} \mathbf{R}_{i, j}$ in (14) depends on the value of $m_{i, j}$ corresponding to $\tau_{i, j}$ and they, respectively, begin from the column of $2 m_{i, j}-1$ and $2 m_{i, j}+1$ for a single DOF system as opposed to the column of $4 m_{i, j}-3$ and $4 m_{i, j}+1$ for a two-DOF one.

Based on (13) and (14), the following mathematical expressions can be established by coupling the solutions of the $k$ successive time intervals in period $T$ :

$$
\mathbf{V}_{k}=\boldsymbol{\Phi} \mathbf{V}_{0}=\mathbf{D}_{k-1} \mathbf{D}_{k-2} \cdots \mathbf{D}_{1} \mathbf{D}_{0} \mathbf{V}_{0},
$$

where $\boldsymbol{\Phi}$ is the Floquet transition matrix that gives the connection between $\mathbf{V}_{k}$ and $\mathbf{V}_{0}$. According to the Floquet theory, the stability of the system is determined using the following criterion. If the moduli of all the eigenvalues of the transition matrix $\Phi$ are less than unity, the system is stable. Otherwise, it is unstable.
Here, it should be noted that the matrix $\mathbf{V}_{i}$ can be reduced because only the delayed positions show up in the governing equation of the milling process. Thus, the size of the approximation vector in (14) could be reduced by removing the delayed values of the velocities, such that the size of vector $\mathbf{V}_{i}$ can be decreased to $M+2$ for a single DOF system and $2 M+4$ for a two-DOF system. This can give some additional improvement in the computational time for the proposed method.

\section{Verification of Method}

There are several numerical and semianalytical techniques to determine the stability conditions for periodic DDEs. However, most of them were developed with the aim of constructing stability charts for milling processes, such as the analysis of the milling system with runout [25], with variable pitch/helix cutter [26-30], with variable-spindle speed [31$33]$, or with serrated cutter $[34,35]$. In order to verify the proposed method, two typical milling operations are chosen and considered. The first is the varying spindle speed process, which can be described by a DDE with time-periodic delay in general. The other is the milling process with variable pitch cutters, which is often characterized by a DDE with multiple delays. Both methods are known means to influence and to prevent chatter vibration in milling.

3.1. Milling with Varying Spindle Speed. Generally, the mathematical models for milling processes with spindle speed variation can be written as

$$
\dot{\mathbf{y}}(t)=\mathbf{A}_{0} \mathbf{y}(t)+\mathbf{A}(t) \mathbf{y}(t)-\mathbf{B}(t) \mathbf{y}(t-\tau(t)) ;
$$

that is, (2) is degenerated into one with one-time-periodic delay. For a single DOF system in [2], the matrices in (16) have the form

$$
\begin{aligned}
\mathbf{A}_{0} & =\left[\begin{array}{cc}
0 & 1 \\
-\omega_{n}^{2} & -2 \zeta \omega_{n}
\end{array}\right], \\
\mathbf{A}(t) & =\mathbf{B}(t)=\left[\begin{array}{cc}
0 & 0 \\
\frac{-G(t)}{m} & 0
\end{array}\right],
\end{aligned}
$$

where $m$ is the mode mass, $\omega_{n}$ is the natural frequency, $\zeta$ is the damping ratios, and $G(t)$ is the specific directional factor and has the form

$$
\begin{aligned}
& G(t) \\
& \quad=a_{p} \sum_{j=1}^{N} g_{j}(t) \sin \phi_{j}(t)\left(K_{t} \cos \phi_{j}(t)+K_{r} \sin \phi_{j}(t)\right),
\end{aligned}
$$

where $a_{p}$ is the axial depth of cut, $N$ is the number of teeth, $K_{t}$ and $K_{r}$ are the linearized cutting coefficients in tangential and radial directions, $g_{j}(t)$ denotes whether the $j$ th tooth is cutting, and the angular position of tooth $j$ is

$$
\phi_{j}(t)=\frac{2 \pi}{60} \int_{0}^{t} \Omega(s) d s+j \frac{2 \pi}{N},
$$




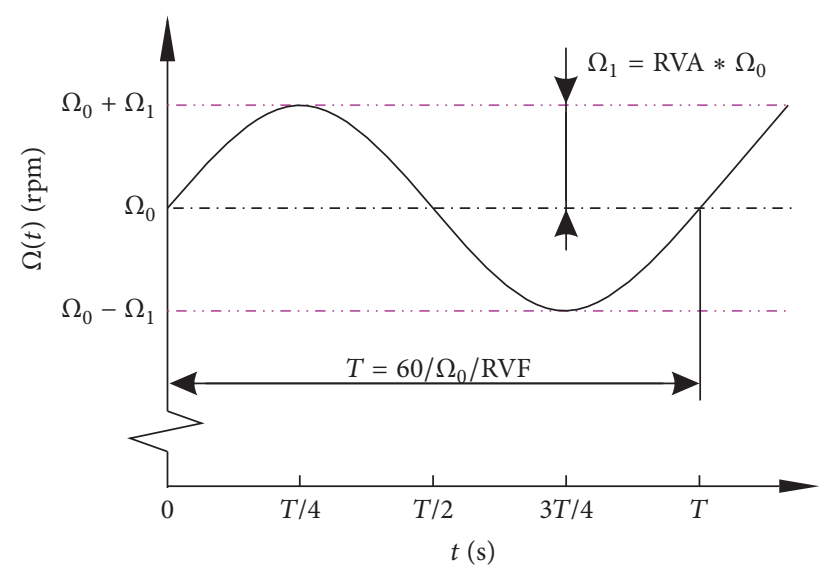

FIGURE 1: Schematic drawing of the sinusoidal modulation of the spindle speed.

where $\Omega(s)$ is the spindle speed and is assumed to change in the form of a sinusoidal wave, which is periodic at a time period $T=60 / \Omega_{0} /$ RVF, with a nominal value, $\Omega_{0}$, and an amplitude $\Omega_{1}=$ RVA $\times \Omega_{0}$, as shown in Figure 1 . For this sinusoidal modulation, the shape function is modeled as

$$
\begin{aligned}
\Omega(t) & =\Omega_{0}+\Omega_{1} \sin \left(\frac{2 \pi}{T} t\right) \\
& =\Omega_{0}\left[1+\mathrm{RVA} \cdot \sin \left(\mathrm{RVF} \cdot \frac{2 \pi}{60} \Omega_{0} t\right)\right],
\end{aligned}
$$

where RVA $=\Omega_{1} / \Omega_{0}$ is the ratio of the speed variation amplitude to the nominal spindle speed and RVF $=60 /\left(\Omega_{0} T\right)$ is the ratio of the speed variation frequency to the nominal spindle speed.

To illustrate the effectiveness of the approach method for milling with spindle speed, the method and results in [2] are taken into consideration. Here, it should be noted that the delayed term is approximated by a linear function of time and the periodic coefficient is approximated by a piecewise constant function for the method in [2]. However, for the proposed method, the delayed term $\mathbf{y}\left(\xi-\tau_{i, j}\right)$, the state term $\mathbf{y}(\xi)$, and the periodic terms $\mathbf{A}(\xi)$ and $\mathbf{C}_{j}(\xi)$ in (7) are all discretized by linear interpolation (see $(8)$ ). Thus, different policies are utilized in the process of equation approximations for two methods. Figure 2 illustrates the stability charts that correspond to the milling processes for RVA $=0.1$ and for four different RVF values using the proposed method and the method in [2]. The parameters are as follows. The cutting processes using a 4 -flute tool $(N=4)$ with zero helix angles are considered under half-immersion up-milling. The cutting force coefficients are $K_{t}=800 \times 10^{6} \mathrm{~N} / \mathrm{m}^{2}$ and $K_{r}=300 \times$ $10^{6} \mathrm{~N} / \mathrm{m}^{2}$. The mode mass is $m=3.1663 \mathrm{~kg}$, the natural frequency is $\omega_{n}=400 \mathrm{~Hz}$, and damping ratios is $\xi=0.02$. It can be seen from Figure 2 that the results obtained via the proposed method in this paper are in close agreement with those in [2].

Meanwhile, the computational times corresponding to every graphic in Figure 2 are also recorded to evaluate the efficiency of proposed method. Here, considering the assumption $q_{1} T=q_{2} \tau_{0}\left(\tau_{0}\right.$ is the tooth passing period) with $q_{1}$ and $q_{2}$ being relatively prime [2] and the equation $q_{1} / q_{2}=\mathrm{RVF} / N$ obtained consequently, if the resolution of $\tau_{0}$ is adopted as 40 and $q_{1}=1$, the resolutions of period $T$ are $320,800,1600$, and 3200 for $\mathrm{RVF}=0.5,0.2,0.1$, and 0.05 , respectively. For a $200 \times 100$ grid of the spindle speed and the depth of cut and a personal computer (Intel(R) Core(TM) i5-2300, 2.8 GHz, $3 \mathrm{~GB}$ ), the computational times are, respectively, $436 \mathrm{~s}, 1026 \mathrm{~s}, 2012 \mathrm{~s}$, and $4132 \mathrm{~s}$ corresponding to RVF = $0.5,0.2,0.1$, and 0.05 for the proposed method as opposed to $1953 \mathrm{~s}, 4757 \mathrm{~s}, 9185 \mathrm{~s}$, and $18341 \mathrm{~s}$ for the method in [2] using our own codes. Time costs reduce nearly by $70 \%$ for every case. Obviously, the low computational cost of our method is illustrated. The reason about the cost reduction can be explained as follows. The matrices $\Phi_{0}, \Phi_{1}, \Phi_{2}$, and $\Phi_{3}$ in (11) are dependent on spindle speed but on depth of cut. Consequently, they are not needed to calculate in the process of sweeping the range of the depth of cut for the proposed method. However, this is also necessary for the method in [2]. Thus, for a parameter plane formed by the spindle speed and the cutting depth and divided into a $N_{s} \times N_{d}$ size grid, the method in [2] must be calculated $N_{s} \times N_{d} \times k$ times to obtain a stability chart, but only $N_{s}$ times for the method in this paper.

3.2. Milling with Variable Pitch Cutter. Considering a system for milling process with variable pitch cutter $[29,30]$ as shown in Figure 3, the mathematical models can be written as

$$
\dot{\mathbf{y}}(t)=\mathbf{A}_{0} \mathbf{y}(t)+\mathbf{A}(t) \mathbf{y}(t)-\sum_{j=1}^{N} \mathbf{B}_{j}(t) \mathbf{y}\left(t-\tau_{j}\right)
$$

that is, the original system of (2) is degenerated into one with multiple delays in this case. $\tau_{j}$ is the pitch period corresponding to the pitch angle $\psi$ (see the right graphic of Figure 3), and the matrices $\mathbf{A}_{0}, \mathbf{A}(t)$, and $\mathbf{B}_{j}(t)$ in (21) have the form

$$
\begin{aligned}
\mathbf{A}_{0} & =\left[\begin{array}{ccccc}
0 & 0 & 1 & 0 & \\
0 & 0 & 0 & 1 & \\
-\omega_{n x}^{2} & 0 & -2 \zeta_{x} \omega_{n x} & 0 & \\
0 & -\omega_{n y}^{2} & 0 & -2 \zeta_{y} \omega_{n y}
\end{array}\right], \\
\mathbf{A}(t) & =\sum_{j=1}^{N} B_{j}(t), \\
\mathbf{B}_{j}(t) & =\left[\begin{array}{cccc}
0 & 0 & 0 & 0 \\
-\frac{a_{p}}{m_{x} h_{x x, j}(t)} & -\frac{a_{p}}{a_{p} h_{x y, j}(t)} & 0 & 0 \\
-\frac{a_{p}}{m_{x} h_{y x, j}(t)} & -\frac{a_{y} h_{y y, j}(t)}{m_{y}} & 0 & 0
\end{array}\right],
\end{aligned}
$$

where $\zeta_{x}$ and $\zeta_{y}$ are the damping ratios, $\omega_{x}$ and $\omega_{y}$ are the natural frequencies, and $m_{x}$ and $m_{y}$ are the modal masses of the cutter. $h_{x x, j}(t), h_{x y, j}(t), h_{y x, j}(t)$, and $h_{y y, j}(t)$ are the cutting force coefficients for the $j$ th tooth defined as 


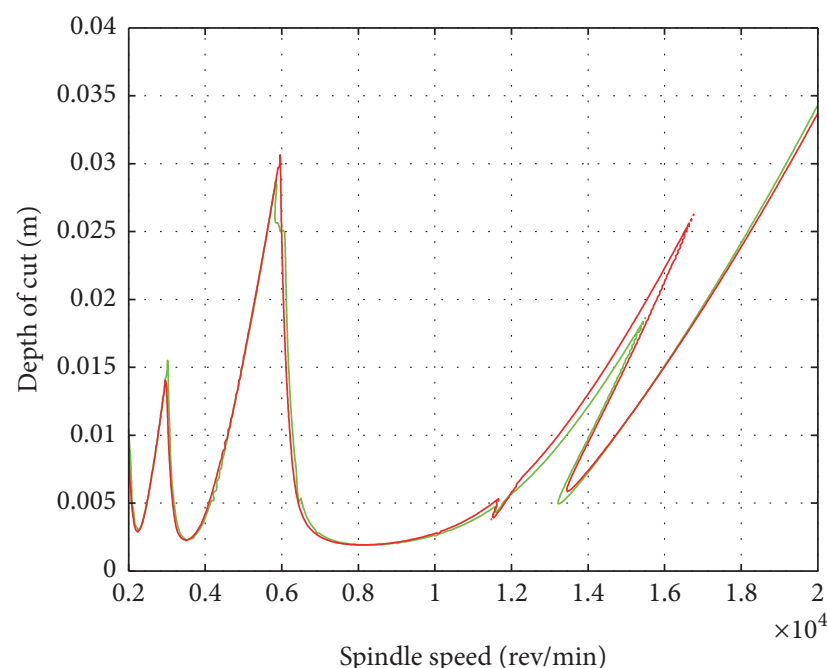

- The method in [2]

The proposed method

(a)

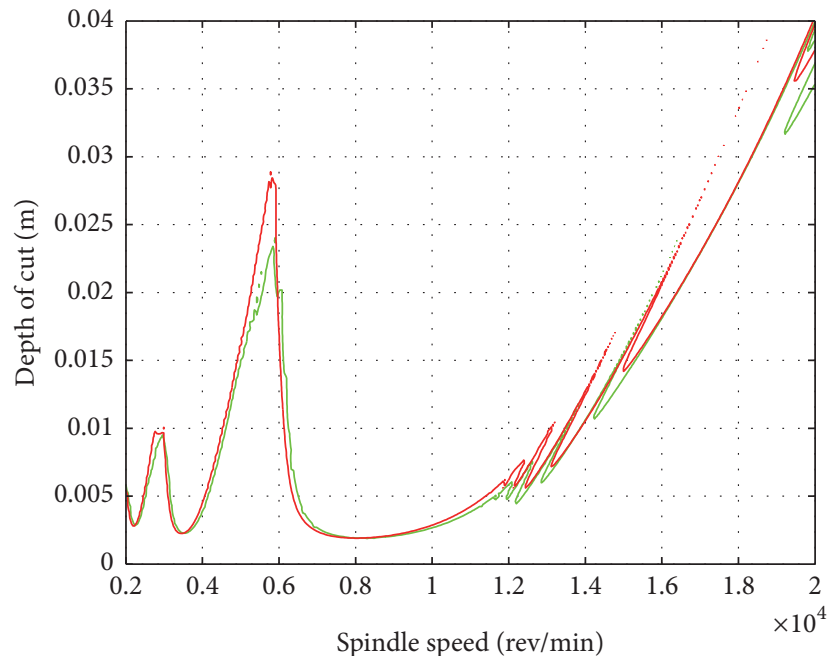

- The method in [2]

— The proposed method

(c)

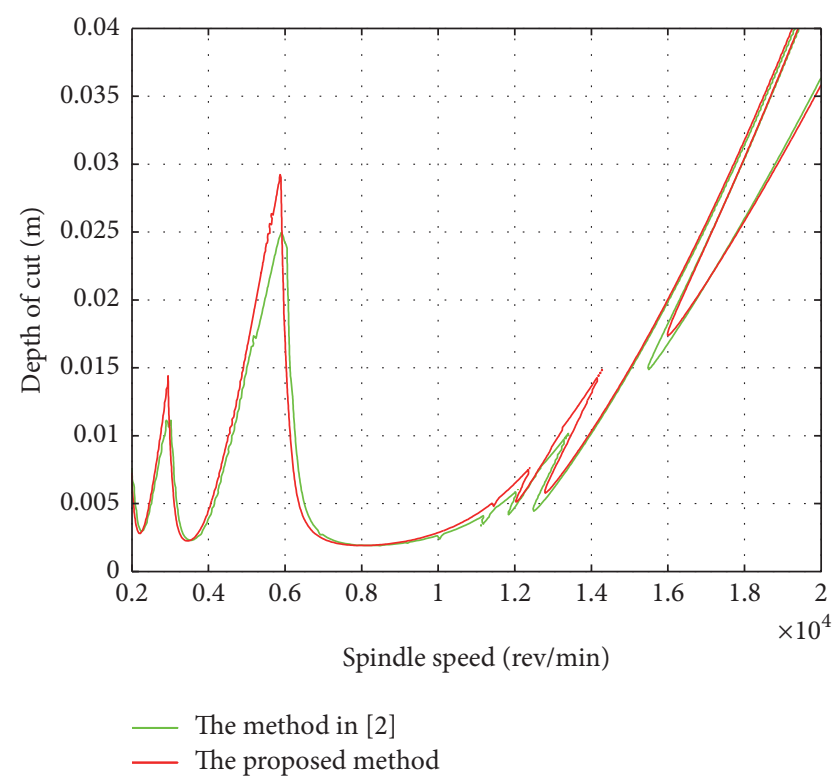

(b)

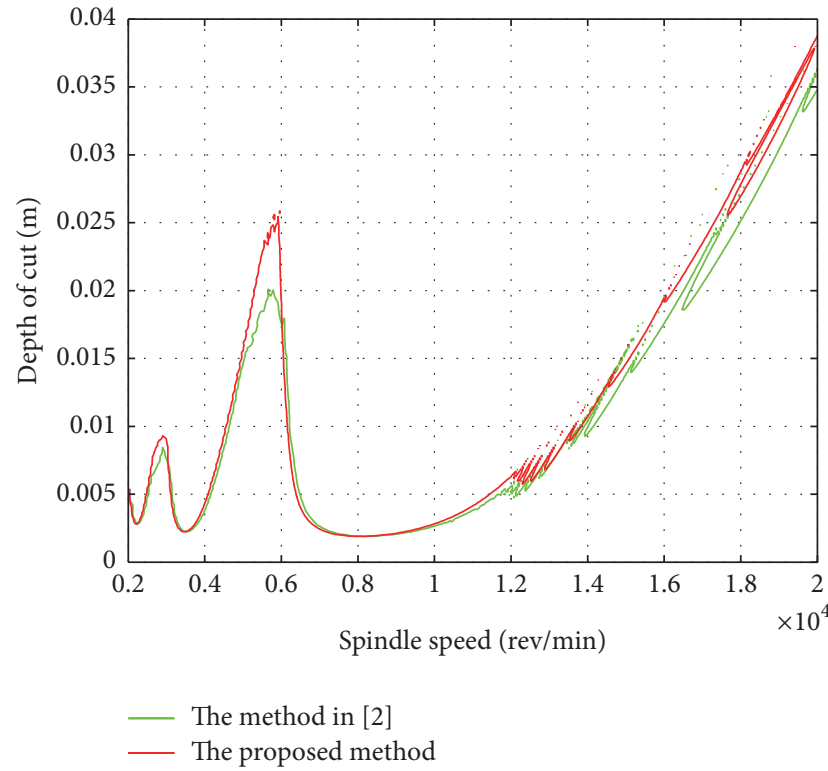

(d)

FIGURE 2: Comparison of stability charts for milling processes with sinusoidal spindle speed modulation with RVA $=0.1$ in the high-speed domain: (a) $\operatorname{RVF}=0.5$; (b) $\mathrm{RVF}=0.2$; (c) $\mathrm{RVF}=0.1$; (d) $\mathrm{RVF}=0.05$.

$$
\begin{aligned}
& h_{x x, j}(t)=g\left(\phi_{j}(t)\right)\left(K_{t} \cos \left(\phi_{j}(t)\right)+K_{r} \sin \left(\phi_{j}(t)\right)\right) \\
& \quad \cdot \sin \left(\phi_{j}(t)\right), \\
& h_{x y, j}(t)=g\left(\phi_{j}(t)\right)\left(K_{t} \cos \left(\phi_{j}(t)\right)+K_{r} \sin \left(\phi_{j}(t)\right)\right) \\
& \quad \cdot \cos \left(\phi_{j}(t)\right), \\
& h_{y x, j}(t)=g\left(\phi_{j}(t)\right) \\
& \cdot\left(-K_{t} \sin \left(\phi_{j}(t)\right)+K_{r} \cos \left(\phi_{j}(t)\right)\right) \sin \left(\phi_{j}(t)\right), \\
& h_{y y, j}(t)=g\left(\phi_{j}(t)\right) \\
& \quad \cdot\left(-K_{t} \sin \left(\phi_{j}(t)\right)+K_{r} \cos \left(\phi_{j}(t)\right)\right) \cos \left(\phi_{j}(t)\right) .
\end{aligned}
$$

To illustrate the performance of the proposed approach on uniform and variable pitch milling tools, the frequencydomain method published in [29] is considered. The comparison of the results using the proposed approach and the method [29] is carried out for both uniform and variable pitch cutter milling, as shown in Figure 4. The main system parameters are down-milling, half-immersion, the number of the cutter teeth which is $N=4$, the natural frequencies which are $\omega_{x}=563.6 \mathrm{~Hz}$ and $\omega_{y}=516.21 \mathrm{~Hz}$, the damping ratios which are $\zeta_{x}=0.0558$ and $\zeta_{y}=0.025$, the modal masses which are $m_{x}=1.4986 \mathrm{~kg}$ and $m_{y}=1.199 \mathrm{~kg}$, and the cutting force coefficients which are $K_{t}=679 \times 10^{6} \mathrm{~N} / \mathrm{m}^{2}$ and 

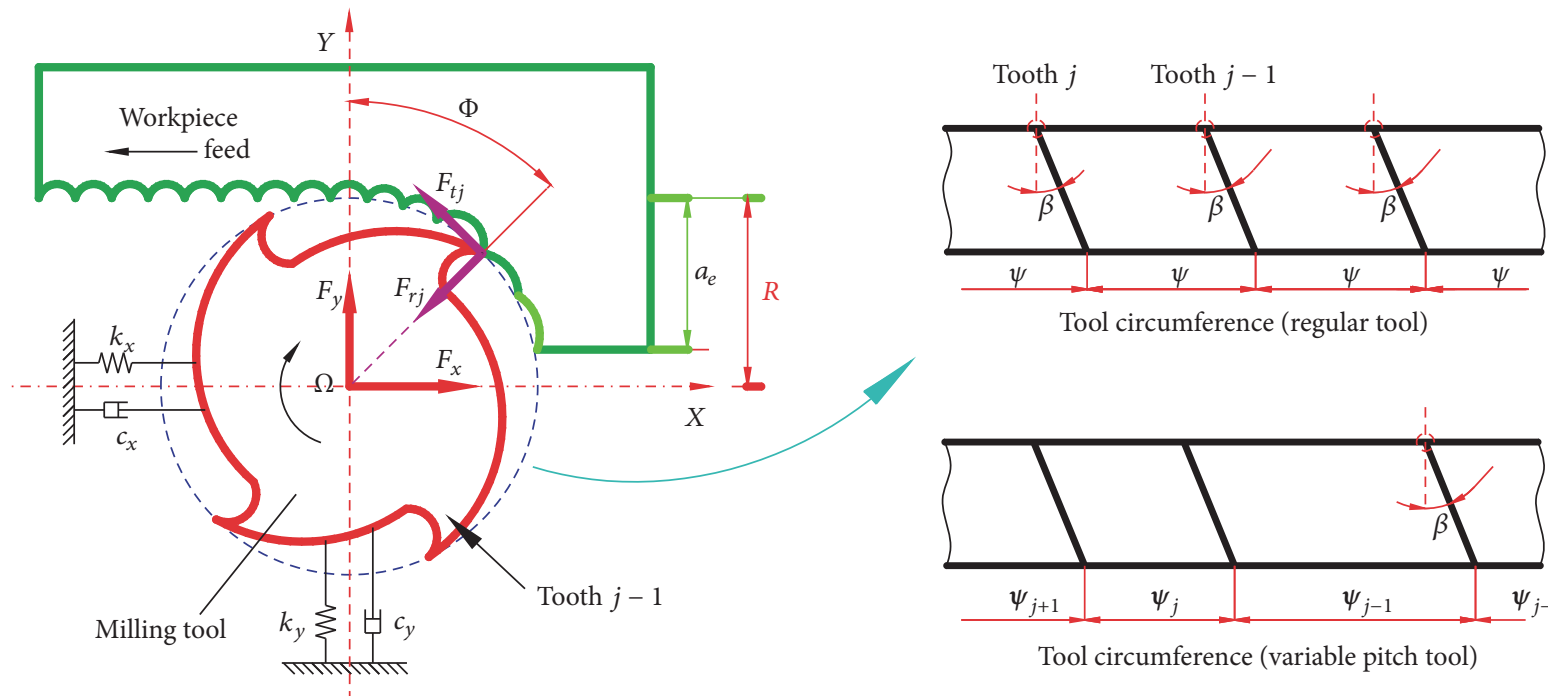

Tool circumference (regular tool)

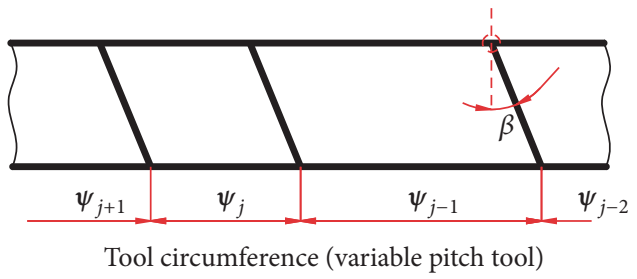

FIGURE 3: Schematic mechanical model of a system for milling process with variable pitch cutter.

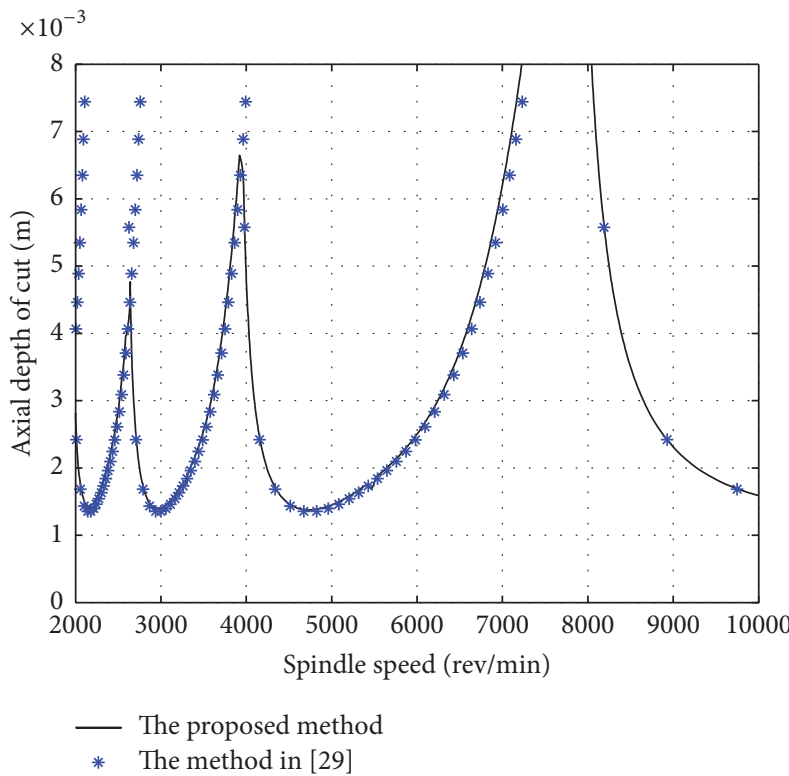

(a)

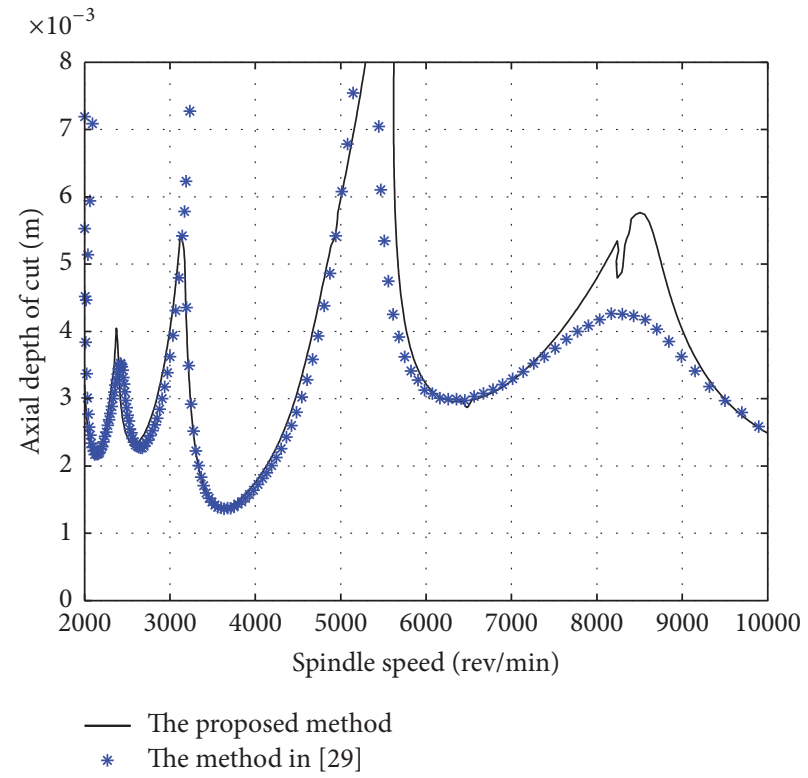

(b)

FIGURE 4: Comparison of the predicted stability lobes: (a) uniform pitch cutter with $\psi=\left[90^{\circ}, 90^{\circ}, 90^{\circ}, 90^{\circ}\right]$ and (b) variable pitch cutter with $\psi=\left[70^{\circ}, 110^{\circ}, 70^{\circ}, 110^{\circ}\right]$.

$K_{t}=256 \times 10^{6} \mathrm{~N} / \mathrm{m}^{2}$. It can be seen from Figure 4(a) that the proposed method agrees closely with the results of analytical method for the uniform pitch cutter milling. For the variable pitch cutter as shown in Figure 4(b), two methods gain consistent predicting results, except for the high-speed domain at approximately $8500 \mathrm{rpm}$, where a clear deviation occurred. Reference [27] chose one point $\left(a_{p}=5 \mathrm{~mm}\right.$ and $\Omega$ $=8500 \mathrm{rpm}$ ) in this deviation and showed its stabilization by time-domain simulations. The reason of this phenomenon is as follows. The proposed method is based on the timeperiodic cutting force coefficients (see (23)), rather than the simplified time-averaged ones in [29]. Thus, the stability prediction by our method is more reasonable and has better accuracy.

\section{Conclusion}

In this work, an improved semidiscretization algorithm is proposed to obtain the stability char for DDEs with multiple time-periodic delays. Two milling examples, variablespindle speed milling system with one-time-periodic delay and variable pitch cutter milling system with multiple delays, 
are utilized to demonstrate effectiveness of the proposed algorithm. Through the comparison with prior works, it is found that the results gained by the presented method in this paper are in close agreement with those existing in the past literature. Moreover, the proposed method also has good computational efficiency. Here, it should be noted that if discussing the milling process only, the proposed method is a generalized algorithm, which can consider the milling processes with variable pitch cutter and variable-spindle speed simultaneously.

\section{Conflicts of Interest}

The authors declare that there are no conflicts of interest regarding the publication of this paper.

\section{Acknowledgments}

This work was supported by the National Natural Science Foundation of China (51405343, 51505336, and 11702192), Tianjin Research Program of Application Foundation and Advanced Technology (15JCQNJC05000 and 15JCQNJC05200), Innovation Team Training Plan of Tianjin Universities and Colleges (TD12-5043), and Tianjin Science and Technology Planning Project (15ZXZNGX00220).

\section{References}

[1] Y. Altintas, Manufacturing Automation, vol. 298, Cambridge University Press, New York, NY, USA, 2012.

[2] T. Insperger and G. Stépán, Semi-Discretization for Time-Delay Systems: Stability and Engineering Applications, vol. 178 of Applied Mathematical Sciences, Springer Science \& Business Media, New York, NY, USA, 2011.

[3] G. Stépán, "Delay, Nonlinear Oscillations and Shimmying Wheels," in Proceedings of the IUTAM Symposium on New Applications of Nonlinear and Chaotic Dynamics in Mechanics, vol. 63, pp. 373-386, Springer, New York, NY, USA.

[4] D. Takács, G. Stépán, and S. J. Hogan, "Isolated large amplitude periodic motions of towed rigid wheels," Nonlinear Dynamics, vol. 52, no. 1-2, pp. 27-34, 2008.

[5] J. Sun, "Delay-dependent stability criteria for time-delay chaotic systems via time-delay feedback control," Chaos, Solitons \& Fractals, vol. 21, no. 1, pp. 143-150, 2004.

[6] J. H. Park and O. M. Kwon, "A novel criterion for delayed feedback control of time-delay chaotic systems," Chaos, Solitons \& Fractals, vol. 23, no. 2, pp. 495-501, 2005.

[7] A. Verdugo and R. Rand, "Hopf bifurcation in a DDE model of gene expression," Communications in Nonlinear Science and Numerical Simulation, vol. 13, no. 2, pp. 235-242, 2008.

[8] A. Ruiz-Herrera, "Chaos in delay differential equations with applications in population dynamics," Discrete and Continuous Dynamical Systems- Series A, vol. 33, no. 4, pp. 1633-1644, 2013.

[9] J. Yan, A. Zhao, and L. Peng, "Oscillation of impulsive delay differential equations and applications to population dynamics," The ANZIAM Journal, vol. 46, no. 4, pp. 545-554, 2005.

[10] S. J. Bhatt and C. S. Hsu, "Stability criteria for second-order dynamical systems with time lag," vol. 33, pp. 113-118, 1966.

[11] E. Budak and Y. Altıntaş, "Analytical prediction of chatter stability in milling, Part I: General formulation," Journal of
Dynamic Systems, Measurement, and Control, vol. 120, pp. 22 30, 1998.

[12] E. Budak and Y. Altıntaş, "Analytical prediction of chatter stability in milling, Part II: application of the general formulation to common milling systems," Journal of Dynamic Systems, Measurement, and Control, vol. 120, no. 1, pp. 31-36, 1998.

[13] S. D. Merdol and Y. Altintas, "Multi frequency solution of chatter stability for low immersion milling," Journal of Manufacturing Science and Engineering, vol. 126, no. 3, pp. 459-466, 2004.

[14] E. A. Butcher and O. A. Bobrenkov, "On the Chebyshev spectral continuous time approximation for constant and periodic delay differential equations," Communications in Nonlinear Science and Numerical Simulation, vol. 16, no. 3, pp. 1541-1554, 2011.

[15] E. A. Butcher, H. Ma, E. Bueler, V. Averina, and Z. Szabo, "Stability of linear time-periodic delay-differential equations via Chebyshev polynomials," International Journal for Numerical Methods in Engineering, vol. 59, no. 7, pp. 895-922, 2004.

[16] T. Insperger and G. Stepan, "Semi-discretization method for delayed systems," International Journal for Numerical Methods in Engineering, vol. 55, no. 5, pp. 503-518, 2002.

[17] T. Insperger and G. Stepan, "Updated semi-discretization method for periodic delay-differential equations with discrete delay," International Journal for Numerical Methods in Engineering, vol. 61, no. 1, pp. 117-141, 2004.

[18] T. Insperger, "Full-discretization and semi-discretization for milling stability prediction: some comments," International Journal of Machine Tools and Manufacture, vol. 50, no. 7, pp. 658-662, 2010.

[19] P. V. Bayly, J. E. Halley, B. P. Mann, and M. A. Davies, "Stability of interrupted cutting by temporal finite element analysis," Transactions of the ASME-Journal of Manufacturing Science and Engineering, vol. 125, no. 2, pp. 220-225, 2003.

[20] Y. Ding, L. Zhu, X. Zhang, and H. Ding, "A full-discretization method for prediction of milling stability," International Journal of Machine Tools and Manufacture, vol. 50, no. 5, pp. 502-509, 2010.

[21] Y. Liu, D. Zhang, and B. Wu, "An efficient full-discretization method for prediction of milling stability," International Journal of Machine Tools and Manufacture, vol. 63, pp. 44-48, 2012.

[22] G. Jin, H. Qi, Y. Cai, and Q. Zhang, "Stability prediction for milling process with multiple delays using an improved semidiscretization method," Mathematical Methods in the Applied Sciences, vol. 39, no. 4, pp. 949-958, 2016.

[23] F. A. Khasawneh and B. P. Mann, "Stability of delay integrodifferential equations using a spectral element method," Mathematical and Computer Modelling, vol. 54, no. 9-10, pp. 24932503, 2011

[24] D. Lehotzky, T. Insperger, and G. Stepan, "Extension of the spectral element method for stability analysis of time-periodic delay-differential equations with multiple and distributed delays," Communications in Nonlinear Science and Numerical Simulation, vol. 35, pp. 177-189, 2016.

[25] M. Wan, W.-H. Zhang, J.-W. Dang, and Y. Yang, "A unified stability prediction method for milling process with multiple delays," International Journal of Machine Tools and Manufacture, vol. 50, no. 1, pp. 29-41, 2010.

[26] N. D. Sims, B. Mann, and S. Huyanan, "Analytical prediction of chatter stability for variable pitch and variable helix milling tools," Journal of Sound and Vibration, vol. 317, pp. 664-686, 2008. 
[27] G. Jin, Q. Zhang, S. Hao, and Q. Xie, "Stability prediction of milling process with variable pitch cutter," Mathematical Problems in Engineering, vol. 2013, Article ID 932013, 2013.

[28] G. Jin, Q. Zhang, S. Hao, and Q. Xie, "Stability prediction of milling process with variable pitch and variable helix cutters," Proceedings of the Institution of Mechanical Engineers, Part C: Journal of Mechanical Engineering Science, vol. 228, no. 2, pp. 281-293, 2014.

[29] Y. Altintaş, S. Engin, and E. Budak, "Analytical stability prediction and design of variable pitch cutters," Transactions of the ASME-Journal of Manufacturing Science and Engineering, vol. 121, no. 2, pp. 173-178, 1999.

[30] G. Jin, Q. Zhang, H. Qi, and B. Yan, "A frequency-domain solution for efficient stability prediction of variable helix cutters milling," Proceedings of the Institution of Mechanical Engineers, Part C: Journal of Mechanical Engineering Science, vol. 228, no. 15, pp. 2702-2710, 2014.

[31] T. Insperger and G. Stéfán, "Stability analysis of turning with periodic spindle speed modulation via semidiscretization," Journal of Vibration and Control, vol. 10, no. 12, pp. 1835-1855, 2004.

[32] M. Zatarain, I. Bediaga, J. Muñoa, and R. Lizarralde, "Stability of milling processes with continuous spindle speed variation: analysis in the frequency and time domains, and experimental correlation," CIRP Annals-Manufacturing Technology, vol. 57, no. 1, pp. 379-384, 2008.

[33] S. Seguy, T. Insperger, L. Arnaud, G. Dessein, and G. Peigné, "On the stability of high-speed milling with spindle speed variation," International Journal of Advanced Manufacturing Technology, vol. 48, no. 9-12, pp. 883-895, 2010.

[34] Z. Dombovari, Y. Altintas, and G. Stepan, "The effect of serration on mechanics and stability of milling cutters," International Journal of Machine Tools and Manufacture, vol. 50, no. 6, pp. 511520, 2010.

[35] Z. Dombovari and G. Stepan, "The effect of helix angle variation on milling stability," Journal of Manufacturing Science and Engineering, Transactions of the ASME, vol. 134, no. 5, Article ID 051015, 2012. 


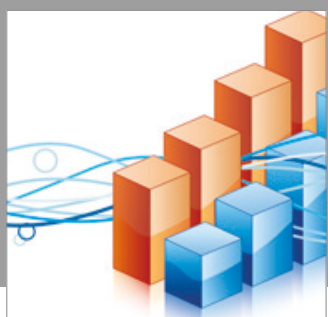

Advances in

Operations Research

vatersals

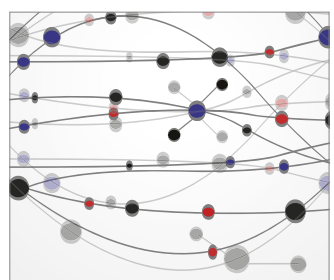

\section{The Scientific} World Journal
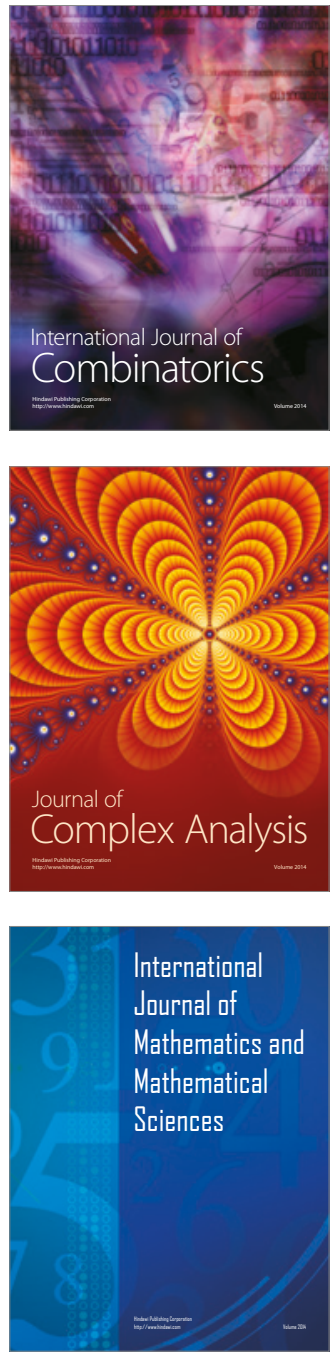
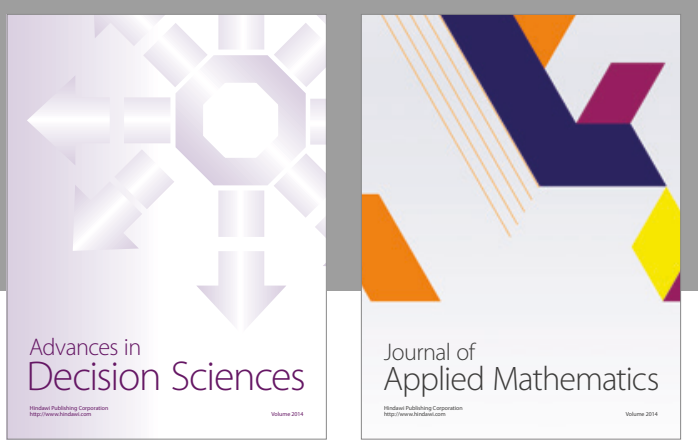

Algebra

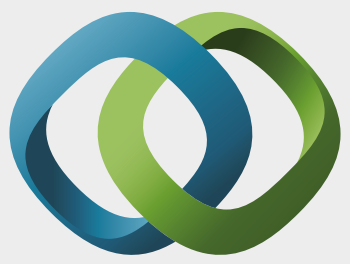

\section{Hindawi}

Submit your manuscripts at

https://www.hindawi.com
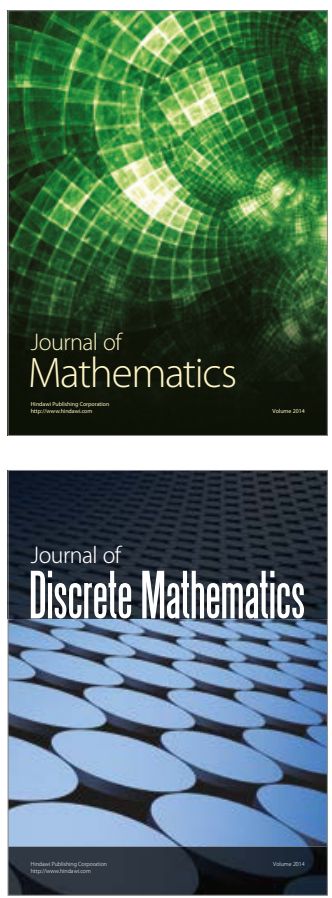

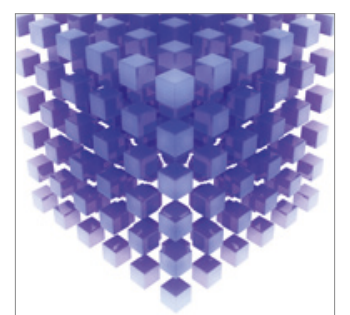

Mathematical Problems in Engineering
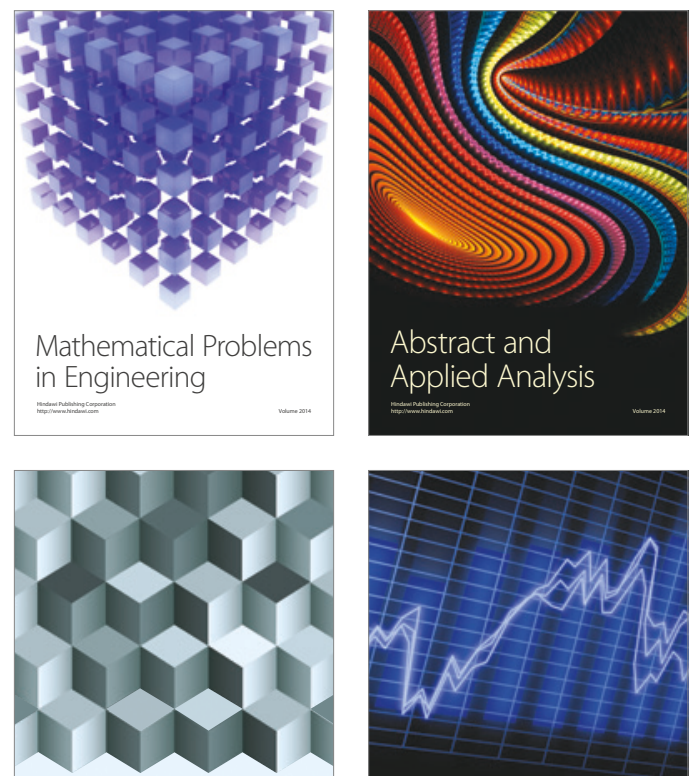

Journal of

Function Spaces

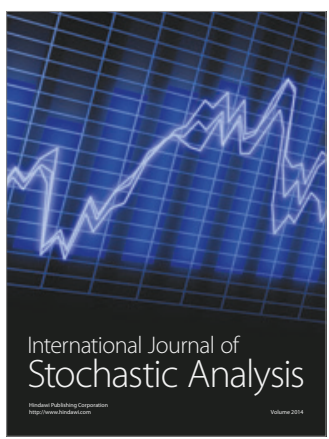

Probability and Statistics
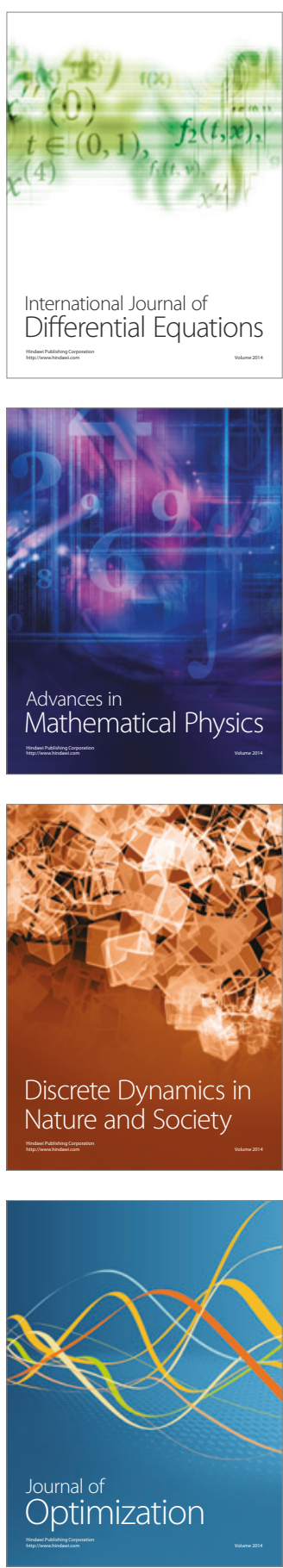